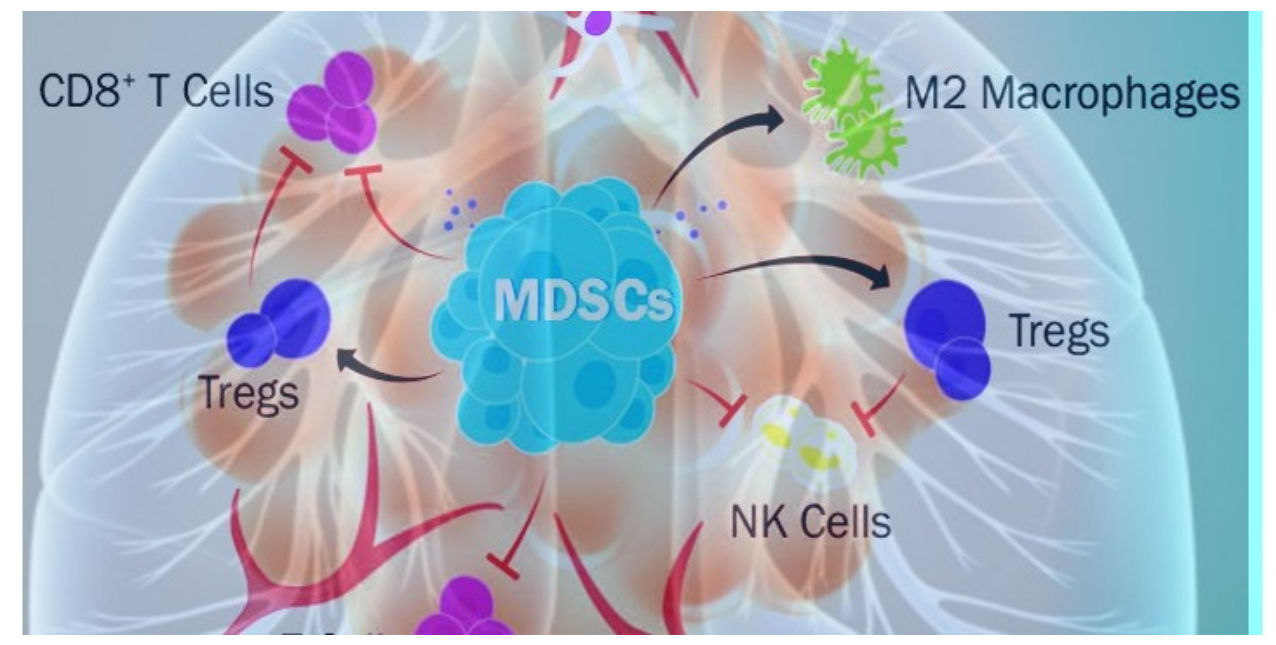

\title{
MDSCs IN COVID-19 Why should we care?
}

\author{
Carlos Rodriguez ${ }^{1}$ \\ 1 Fundación Jiménez Díaz
}

Funding: The author(s) received no specific funding for this work.

Potential competing interests: The author(s) declared that no potential competing interests exist.

\section{Abstract}

For the last few months, we all, scientists and industry alike, have been working in a rush to try to understand the mechanics of the SARS-CoV2 coronavirus, the cause of the Covid-19 disease. Efforts have been particularly centered on finding ways to predict the bad clinical evolution that sometimes occur in patients with minimal respiratory symptoms at presentation. We know already that most of these patients do not progress to more severe forms of the disease. There is however a small percentage of patients that develop what is called Severe Acute Respirtatory Syndrome or SARS, which is the cause of death in this disease.

SARS (or more generally speaking ARDS) is caused by a massive unordered reaction of the immune system in the lungs. We have found some data in the recent literature indicating the presence of a type of cells called Myeloid Derived Suppressor Cells (MDSCS) over the course of the disease. These cells have been previously studied for their major role in nurturing cancer growth. After a careful review of the litterature, now we believe that MDSCS may also have a major role in SARS progression and, in the survivors, they could be responsible for post-Covid-19 health conditions long time 
after recovery from the acute phase of the disease. If our premises are confirmed, we think that these cells should be priority targets for the design of predictive methods based on their early detection and quantitation. MDSCs should also be the focus of investigation in search for candidate drugs that could inhibit their development in the bone marrow, their migration into the lungs, or their suppressive regulation of $\mathrm{T}$ cell activity during the viral infection and beyond.

Corresponding Author: Carlos Rodríguez, Paseo Imperial Health Center, 28005 Madrid+34650311449 nebibatus@gmail.com

\section{Introduction}

Since the first cases of pneumonia were diagnosed in Wuhan, China, in Dec 2019 $9^{[1][2][3],}$ the new SARS-CoV2 coronavirus has taken hold of the entire world in the form of a pandemic with hundreds of thousands deaths and, specially, the growing uncertainty associated with the absence of an end in sight to it. Despite all this, the Covid-19 disease has also brought positives amongst all the havoc. A case in point: both the scientific community and the industry are joining forces in an almost unprecedented approach to work together for the common goal of being able to stop the death count.

In our view, this common front should aim at rapidly improving, at least, three main aspects of the disease: 1) The early detection of patients at risk of developing SARS, 2) The early treatment to prevent lethality 3) The appraisal of the post-recovery long-term health consequences in survivors.

As we will discuss in the coming pages, the key for a successful strategy against the Covid-19 disease consists in developing tools (both diagnostic and therapeutic) that can be deployed during the very early phases of the disease. With this, we refer to the first flu-like symptoms of the disease. When these first symptoms are present in people at risk of developing SARS (elderly people or patients with previous conditions such as hypertension, diabetes, ischemic heart disease, cancer or $\mathrm{COPD}^{[4]}$ ), we should be able to detect early signs of deterioration and, consequently, apply the according therapeutic measures to prevent further worsening. In this regard, we have already proposed a treatment for this budding phase of the disease. The treatment consists in an adaptation 
of the scheduled inhalers used in the chronic treatment of asthma and this approach is predicted to slow down or stop the progression of the disease in this group of patients at risk $^{[5]}$.

The early phases of the Covid-19 disease are usually spotted at home, at a primary care center or at the hospital ER, where patients look for a first health assessment of their symptoms (cough, diarrhea, abdominal discomfort, chest tightness, dysgeusia, etc.). At this point, we would like to be able to decide if a patient is really at risk of quickly progressing towards a more severe form and in this case, he/she should be transferred to the ward or referred to the closest hospital for further evaluation and treatment, or, if, on the contrary, we could wait to take further measures.

To sum up, we are in serious need of appropriate tools to precisely assess on the spot the risk of developing the severe complications in suspected or confirmed Covid-19 patients .

\section{Covid-19 Disease}

The Covid-19 disease as we know it is in fact two diseases in one. Most of the people infected by the SARS-CoV2 coronavirus are either asymptomatic throughout the entire course of the disease or have more or less mild symptoms followed by an apparently full recovery ${ }^{[6]}$. In those patients, the infection behaves more or less like a classical respiratory viral illness. In a minority of infected people, however (around 10\%), the symptoms (particularly the respiratory symptoms) are more severe and, thus, the disease can evolve into what is called an Acute Respiratory Distress Syndrome (ARDS or SARS). ARDS is often an irreversible process that can lead to death (most deaths following Covid-19 are due to ARDS) or, in survivors, to long term yet uncharacterized chronic health conditions ${ }^{[7]}$.

The scientific community has reached a consensus with respect to the underlying major mechanism of the disease. It appears that the cause of the sudden clinical deterioration that occurs in a minority of patients with Covid-19 is due to an overreaction of the immune system within the lungs in the form of a so-called "cytokine storm"[8][9]. This aberrant reaction is most likely due to the uncontrolled response of the pulmonary innate immune system to the CoV2 coronavirus infection ${ }^{[10][11]}$. On the one hand, on the march leading to ARDS, there is an overabundance of pro-inflammatory cytokines that flood into the lungs and, on the other hand, there is a striking absence of the major control component of the immune system: T cells. These cells in all of their forms and 
shapes (CD4+ Helper, CD8+ Cytotoxic, Tregs or NK cells) are either dramatically reduced, exhausted, or inactive in the context of ARDS (and this is often translated into a global overall sharp decrease in lymphocyte count in the peripheral blood of these patients ${ }^{[12]}$ ). In other words, they are unable to orchestrate an ordered sequential adaptive immune response to the coronavirus infection. Instead, during the early phases of ARDS, we witness a runaway chain reaction of the immune system with a plethora of proinflammatory cytokines and chemokines, that either destroy the lung tissue (leading to liquid occupance of the alveoli) or attract effector cells like monocytes or neutrophils with similar destructive power.

\section{Main}

One of the reasons behind the apparent innaction of the adaptive immune system (mainly T and B cells) is the SARS-CoV2 virus itself. The genome of this virus encodes several proteins that have precisely this function (to anesthetize the adaptive immune system by blocking for instance the type I interferon response ${ }^{[13]}$. Another reason, and the one that is most relevant to this paper, is linked to the kind of cells that, coming from the Bone Marrow, are attracted to the site of the infection in the alveolar and perialveolar lung space.

Cells that are attracted to the lungs during SARS are mainly monocytes (the precursors of non-resident macrophages as well as of dendritic cells) and granulocytes (PMN). But, as we will see, not any type of monocytes or granulocytes.

Classical monocytes are characterized by a pattern of expression of surface proteins $\left(\mathrm{CD} 14^{\text {hi }} \mathrm{CD} 16^{-}\right)$that facilitate their purification by flow cytometry ${ }^{[14]}$. During SARS however it has been shown that there is a sharp decrease in this subpopulation of classical monocytes and instead atypical monocytes with the signature CD14 ${ }^{\text {low } H L A D R}{ }^{\text {low }} C D 16^{\text {hi }}$ are abundantly present in the peripheral blood. Moreover, this population can be accurately quantified and it positively correlates with disease severity

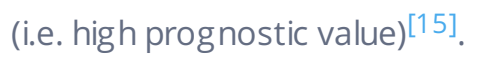

Also in the lungs of patients with Covid-19, a subpopulation of atypical neutrophils, the so-called Low Density Granulocytes (LDG), has been identified and are likely to have a decisive function in the fate of the disease ${ }^{[16]}$.

Indeed, low density granulocytes have been recently identified in patients with Chronic Critical IIIness (CCI), 'a persistent inflammatory-immunosuppressive and catabolic 
syndrome ${ }^{[17]}$. They are also overrepresented during septic shock and here their presence is directly correlated with bad prognosis ${ }^{[18]}$.

\section{Myeloid Derived Suppressor Cells (MDSCs)}

These atypical forms of myeloid cells have been recently grouped together with other types of peripheral mononuclear cells under the provisional classificatory umbrella of Myeloid Derived Suppressor Cells or MDSC ${ }^{[19]}$. MDSCs are defined as immature myeloid cells that egress from the bone marrow in different disease contexts. What all these cells have in common is their capacity to suppress the adaptive immune system ${ }^{[20]}$. Indeed, MDSCs inhibit adaptive antitumor immunity by inhibiting $T$ cell activation and function ( $T$ cell receptor downregulation, T-cell cycle inhibition and immune checkpoint blockade) ${ }^{[21]}$, and by driving and recruiting T regulatory cells. Immunosuppression by MDSCs is also mediated by the generation of reactive oxygen species (ROS) ${ }^{[22]}$ and cytokine release (IL10$, T GF- $\beta)^{[23]}$.

Two different subtypes of MDSCs, monocytic MDSCs and granulocytic MDSCs (mMDSCs and gMDSCs), have been identified ${ }^{[24]}$ and the expansion and activation of MDSCs have been shown to be trigg ered by a number of cell-derived factors. For instance, granulocyte-colony-stimulating factor (G-CSF) has been identified as a major factor for the differentiation of $\operatorname{gMDSCS}^{[25]}$, and granulocyte-macrophage colonystimulating factor (GM-CSF) has been shown to play a key role in mMDSC production ${ }^{[26]}$.

Sure enough, both G-CSF and GM-CSF are among the cytokines that are produced in excess in the lungs of SARS patients, implying that they are probably the necessary cues for the differentiation of both M and G-MDSC and their migration from the bone marrow into the inflamed lungs ${ }^{[27][28]}$. Not less important, a major chemoattractant for neutrophils (the CXCL-8 chemokine) is also one of the higher expressing cytokines during $\mathrm{SARS}^{[29]}$ and most of the chemokines known to attract monocytes are produced in abundance in the lungs of Covid-19 patients. All of these factors are exceedingly spilled out of the lungs into the circulation ${ }^{[30]}$. In a nutshell, the prerequisites for the production of MDSC and their massive migration into the lungs are clearly fulfilled by the cytokine storm taking place within the lungs of these patients.

MDSCs have been thoroughly characterized in several autoimmune diseases like SLE ${ }^{[31]}$, $\mathrm{DMI}^{[32]}$, in severe sepsis ${ }^{[33]}$, where their prominence is linked to disease severity, as well as in different tumor models, where they are consistently involved in promoting tumor growth $^{[34]}$. 
This latter aspect has a potential important bearing on Covid-19. Indeed, cancer has been consistently placed along the list of health preconditions with higher risk of developing $\mathrm{SARS}^{[35]}{ }^{[36]}$. More specifically, the elevated risk of death is correlated with active cancer and not with its current treatment at the time of contracting Covid-19 ${ }^{[37][38]}$. As we know, active cancer is precisely when MDSC are at their peak both in terms of number and functional activation ${ }^{[39]}$.

Particularly relevant is also the role of MDSC in a plethora of viral infections ${ }^{[40]}$. For instance, during infection with the HCV (A +ssRNA virus like the CoV2 coronavirus), CD4+, CD8+ and NK cell functions are abrog ated by a prominent population of MDSC, thus promoting viral replication and disease chronicity ${ }^{[41][42]}$. Also M-MDSC with immunosuppressive activity have been isolated from the blood of humans and mice infected with another RNA virus (the influenza A virus or IAV) ${ }^{[43]}$. Finally, the effect of both G-MDSC and M-MDSC on anti-viral immunity has been amply studied in HIV infected patients and animal models, showing that mechanisms of immune-suppression can be attributed to inducible NO synthase (iNOS), Arginase-1 (Arg-1), or the generation of Reactive Oxyg en Species (ROS) by associated MDSCs ${ }^{[44][45]}$.

Importantly, and paradoxically, MDSC (both G and M-MDSC) show strong features of proinflammatory cells. In fact, upon activation, they can injure endothelial cells and release a large quantity of tumor necrosis factors (TNFs) and type I and type II interferons (IFNs) ${ }^{[46]}$. In the case of neutrophils, this effect has been linked to an excess of so-called neutrophil extracellular traps (NETs) that are released from neutrophils during inflammation. NETs are webs of extracellular DNA decorated with histones, myeloperoxidase, and elastase ${ }^{[47]}$. Although NETs contribute to pathogen clearance, excessive NET formation, as observed after massive tissue damage, promotes inflammation and tissue damage in sepsis as well as in macroscopic tissue injury ${ }^{[48]}$. NETs have been extensively studied in the context of postinflammatory thrombosis as observed in the Covid-19 disease ${ }^{[49]}$ and the contribution of NETs to coagulation and platelet aggregation (and the elevation of molecular markers like the D-Dimer) has been extensively studied ${ }^{[50]}$. Therefore, the importance of NETs in SARS cannot be ignored anymore, to the point that it has been recently postulated that their abundance could be intimately correlated with the clinical deterioration seen with Covid-19 ${ }^{[51]}$. Since NETs are the consequence of both tissue damage and the presence of proinflammatory neutrophils such as the LDG as present in SARS, we foresee that the framework of this paper could also be applied to predict the occurrence of pro-coagulation events such as 
the thrombosis and ischemic heart and brain complications that are often observed in the severe course of the disease.

\section{Long term effects of immunosuppression by MDSC}

A very prominent aspect, and also a very important one for this layout, is the long term effects of MDSC. It has been extensively reported that the appearance of MDSC is not an isolated/reversible event. Indeed, After Severe Sepsis/Septic Shock in humans, circulating MDSCs are persistently increased, functionally immunosuppressive, and associated with adverse outcomes ${ }^{[52]}$. This long term phenomenon has also been reported in rat models after Trauma and Hemorrhagic Shock ${ }^{[53]}$. The phenomenon is so important that the term sepsis-acquired immunodeficiency has been recently coined ${ }^{[54]}$. There is now increasing evidence that acquired immune dysfunctions might contribute to the increased susceptibility to secondary infections or autoimmune disorders in the wake of recovery after a long ICU stay ${ }^{[55]}$.T hat there is a permanent modification after sepsis, has been demonstrated using a molecular approach and it has been linked to specific epigenetic changes ${ }^{[56]}$. There are even candidate drugs like LDK378 (an inhibitor of the ALK kinase) that prevents the recruitment of MDSCs to spleen via the CCR2 pathway in mice with sepsis ${ }^{[57]}$.

To our knowledge, a similar long-term immunosuppression after recovery from viral infections has not been reported yet. We attribute the absence of studies of this kind to the fact that the interest in these cells in relation with acute or chronic viral infections is only very young (perhaps limited to the last three years). This lack of data, particularly in the Covid-19 context, should encourage scientists around the world to delve into the issue. That will help us all to better understand the disease and, perhaps, to come up with new urgently needed solutions therefor.

To recapitulate, there are some experimental observations that indicate the presence of MDSC in the lung (the epicenter of SARS) of Covid-19 patients. We believe that these cells may have a pivotal role in the unordered immune response that is at the core of SARS. We predict that the presence of MDSCs will be relevant even after recovery from the acute disease and could be cause of yet unknown chronic health conditions post-Covid19. We finally believe that the MDSC cells should be analyzed and characterized in the Covid-19 disease, and we foresee a plethora of new insights, diagnostics and treatments that will come out of such endeavor. 


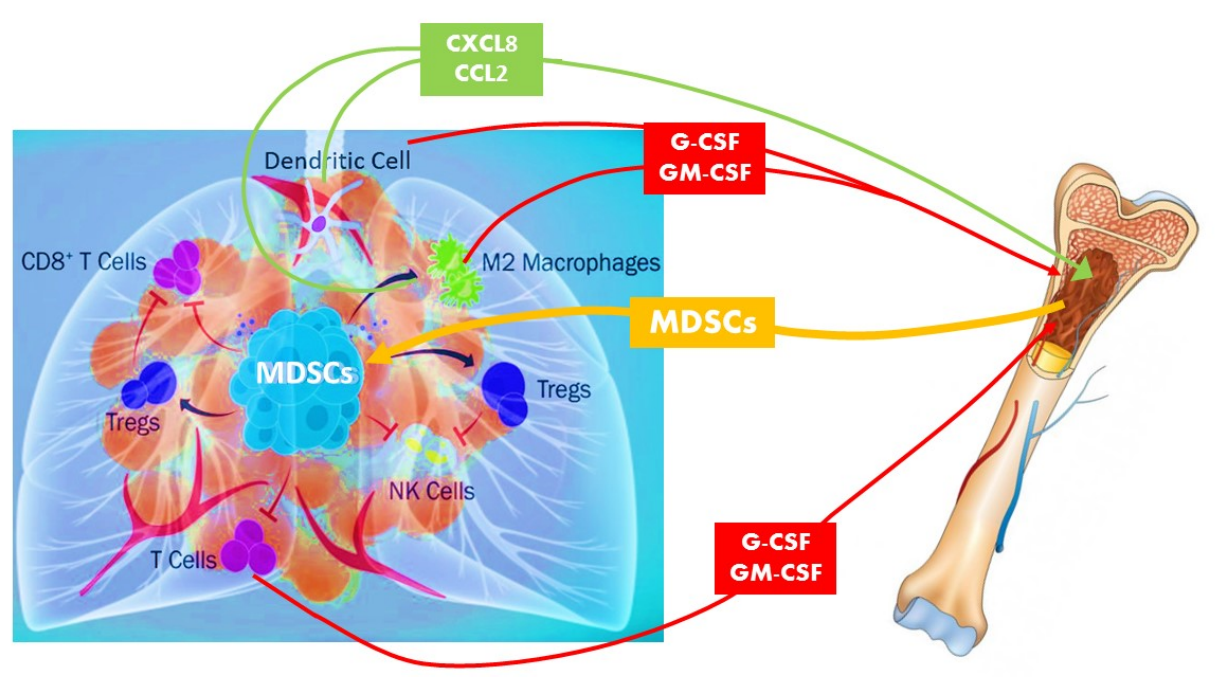

Figure 1

Adapted from https://www.rndsystems.com/

References

1. ^ Bruce Spiess. (2020). Faculty Opinions recommendation of Clinical features of patients infected with 2019 novel coronavirus in Wuhan, China.. doi:10.3410/f.737258313.793572876.

2. `Chen Wang, Peter W Horby, Frederick G Hayden, George F Gao. (2020). A novel coronavirus outbreak of global health concern. The Lancet, vol. 395 (10223), 470-473. doi:10.1016/s0140-6736(20)30185-9.

3. ^ Na Zhu, Dingyu Zhang, Wenling Wang, Xingwang Li, Bo Yang, Jingdong Song. (2020). A Novel Coronavirus from Patients with Pneumonia in China, 2019. N EnglJ Med, vol. 382 (8), 727-733. doi:10.1056/nejmoa2001017.

4. ' Damian N Valencia. (2020). Brief Review on COVID-19: The 2020 Pandemic Caused by SARS-CoV-2. doi:10.7759/cureus.7386.

5. ^Carlos Rodriguez, Carlos Veciana. THE COMBINATION OFINHALED BUDESONIDE AND FORMOTEROL AS AN EARLY TREATMENT FOR THE COVID-19 DISEASE.

6. ^Monica Gandhi, Deborah S. Yokoe, Diane V. Havlir. (2020). Asymptomatic Transmission, the Achilles' Heel of Current Strategies to Control Covid-19. N EnglJ Med, vol. 382 (22), 2158-2160. doi:10.1056/nejme2009758.

7. ^ B. Taylor Thompson, Rachel C. Chambers, Kathleen D. Liu. (2017). Acute Respiratory Distress Syndrome. N EnglJ Med, vol. 377 (6), 562-572. doi:10.1056/nejmra1608077.

8. ^ Francesca Coperchini, Luca Chiovato, Laura Croce, Flavia Magri, Mario Rotondi. (2020). The cytokine storm in COVID-19: An overview of the involvement of the chemokine/chemokine-receptor system. Cytokine \& Growth Factor Reviews. 
doi:10.1016/j.cytogfr.2020.05.003.

9. ^ Wasco Wruck, James Adjaye. (2020). Meta-analysis of transcriptomes of SARS-Cov2 infected human lung epithelial cells identifies transmembrane serine proteases coexpressed with ACE2 and biological processes related to viral entry, immunity, inflammation and cellular stress. doi:10.1101/2020.05.12.091314.

10. ^ Klara Kubelkova, Ales Macela. (2019). Innate Immune Recognition: An Issue More Complex Than Expected. Front. Cell. Infect. Microbiol., vol. 9. doi:10.3389/fcimb.2019.00241.

11. ^ Masahiro Yamamoto, Shintaro Sato, Hiroaki Hemmi, Satoshi Uematsu, Katsuaki Hoshino, Tsuneyasu Kaisho. (2003). TRAM is specifically involved in the Toll-like receptor 4-mediated MyD88-independent signaling pathway. Nat Immunol, vol. 4 (11), 1144-1150. doi:10.1038/ni986.

12. ^ Moritz Anft, Krystallenia Paniskaki, Arturo Blazquez-Navarro, Adrian Atila Nicolas Doevelaar, Felix Seibert, Bodo Hoelzer. (2020). COVID-19 progression is potentially driven by $T$ cell immunopathogenesis. doi:10.1101/2020.04.28.20083089.

13. ^Helen K. W. Law, Chung Yan Cheung, Hoi Yee Ng, Sin Fun Sia, Yuk On Chan, Winsie Luk. (2005). Chemokine up-regulation in SARS-coronavirus-infected, monocytederived human dendritic cells. doi:10.1182/blood-2004-10-4166.

14. ^ Theodore S. Kapellos, Lorenzo Bonaguro, Ioanna Gemünd, Nico Reusch, Adem Saglam, Emily R. Hinkley. (2019). Human Monocyte Subsets and Phenotypes in Major Chronic Inflammatory Diseases. Front. Immunol., vol. 10. doi:10.3389/fimmu.2019.02035.

15. ^ANDREA LOMBARDI, Elena Trombetta, Alessandra Cattaneo, Valeria Castelli, Emanuele Palomba, Mario Tirone. (2020). Early phases of COVID-19 are characterized by a reduction of lymphocyte populations and the presence of atypical monocytes. doi:10.1101/2020.05.01.20087080.

16. ^Andrea R Daamen, Prathyusha Bachali, Katherine A Owen, Kathryn M Kingsmore, Erika L Hubbard, Adam C Labonte. (2020). Comprehensive Transcriptomic Analysis of COVID-19 Blood, Lung, and Airway. doi:10.1101/2020.05.28.121889.

17. `Juan C. Mira, Lori F. Gentile, Brittany J. Mathias, Philip A. Efron, Scott C. Brakenridge, Alicia M. Mohr. (2017). Sepsis Pathophysiology, Chronic Critical Illness, and Persistent Inflammation-Immunosuppression and Catabolism Syndrome. Critical Care Medicine, vol. 45 (2), 253-262. doi:10.1097/ccm.0000000000002074.

18. ^ Fabrice Uhel, Imane Azzaoui, Murielle Grégoire, Céline Pangault, Joelle Dulong, JeanMarc Tadié. (2017). Early Expansion of Circulating Granulocytic Myeloid-derived Suppressor Cells Predicts Development of Nosocomial Infections in Patients with Sepsis. Am J Respir Crit Care Med, vol. 196 (3), 315-327. doi:10.1164/rccm.201606- 
$11430 c$.

19. ^ Peiwen Ma, Pamela L. Beatty, John McKolanis, Randal Brand, Robert E. Schoen, Olivera J. Finn. (2019). Circulating Myeloid Derived Suppressor Cells (MDSC) That Accumulate in Premalignancy Share Phenotypic and Functional Characteristics With MDSC in Cancer. Front. Immunol., vol. 10 . doi:10.3389/fimmu.2019.01401.

20. ^Mohammad H. Rashid, Thaiz F. Borin, Roxan Ara, Raziye Piranlioglu, Bhagelu R. Achyut, Hasan Korkaya. (2020). The critical immunosuppressive effect of MDSCderived exosomes in the tumor microenvironment. doi:10.1101/2020.03.05.979195.

21. `Zhenzhen Yang, Jiacheng Guo, Lanling Weng, Wenxue Tang, Shuiling Jin, Wang Ma. (2020). Myeloid-derived suppressor cells - new and exciting players in lung cancer.J Hematol Oncol, vol. 13(1). doi:10.1186/s13045-020-0843-1.

22. ^ Cesar A. Corzo, MatthewJ. Cotter, Pingyan Cheng, Fendong Cheng, Sergei Kusmartsev, Eduardo Sotomayor. (2009). Mechanism Regulating Reactive Oxygen Species in Tumor-Induced Myeloid-Derived Suppressor Cells. J Immunol, vol. 182 (9), 5693-5701. doi:10.4049/jimmunol.0900092.

23. `Jun Dai, Mohamed El Gazzar, Guang Y. Li, Jonathan P. Moorman, Zhi Q. Yao. (2014). Myeloid-Derived Suppressor Cells: Paradoxical Roles in Infection and Immunity. J Innate Immun, vol. 7 (2), 116-126. doi:10.1159/000368233.

24. `Elisa Peranzoni, Serena Zilio, Ilaria Marigo, Luigi Dolcetti, Paola Zanovello, Susanna Mandruzzato. (2010). Myeloid-derived suppressor cell heterogeneity and subset definition. Current Opinion in Immunology, vol. 22 (2), 238-244. doi:10.1016/j.coi.2010.01.021.

25. `M. Kowanetz, X. Wu, J. Lee, M. Tan, T. Hagenbeek, X. Qu. (2010). Granulocyte-colony stimulating factor promotes lung metastasis through mobilization of $L y 6 G+L y 6 C+$ granulocytes. Proceedings of the National Academy of Sciences, vol. 107 (50), 2124821255. doi:10.1073/pnas.1015855107.

26. ^Lauren J. Bayne, Gregory L. Beatty, Nirag Jhala, Carolyn E. Clark, Andrew D. Rhim, Ben Z. Stanger. (2012). Tumor-Derived Granulocyte-Macrophage Colony-Stimulating Factor Regulates Myeloid Inflammation and T Cell Immunity in Pancreatic Cancer. Cancer Cell, vol. 21 (6), 822-835. doi:10.1016/j.ccr.2012.04.025.

27. ^Y Yonggang Zhou, Binqing Fu, Xiaohu Zheng, Dongsheng Wang, Changcheng Zhao, Yingjie Qi. (2020). Pathogenic T-cells and inflammatory monocytes incite inflammatory storms in severe COVID-19 patients. doi:10.1093/nsr/nwaa041.

28. ^ Dandan Wu, Xuexian O. Yang. (2020). TH17 responses in cytokine storm of COVID19: An emerging target of JAK2 inhibitor Fedratinib. Jo urnal of Microbiology, Immunology and Infection. doi:10.1016/j.jmii.2020.03.005.

29. ^ Georg Schett, Michael Sticherling, Markus F. Neurath. (2020). COVID-19: risk for 
cytokine targeting in chronic inflammatory diseases? Nat Rev Immunol, vol. 20 (5), 271-272. doi:10.1038/s41577-020-0312-7.

30. ^Xiaowei Li, Manman Geng, Yizhao Peng, Liesu Meng, Shemin Lu. (2020). Molecular immune pathogenesis and diagnosis of COVID-19. Journal of Pharmaceutical Analysis, vol. 10 (2), 102-108. doi:10.1016/j.jpha.2020.03.001.

31. `Jianjian Ji, Pengfei Li, Cunsi Shen, Huan Dou, Tingting Wang, Liyun Shi. (2019). MDSCS: friend or foe in systemic lupus erythematosus. Cell Mol Immunol, vol. 16 (12), 937-939. doi:10.1038/s41423-019-0271-8.

32. ^ Fatima Whitfield-Larry, Jamie Felton, John Buse, Maureen A. Su. (2014). Myeloidderived suppressor cells are increased in frequency but not maximally suppressive in peripheral blood of Type 1 Diabetes Mellitus patients. Clinical Immunology, vol. 153 (1), 156-164. doi:10.1016/j.clim.2014.04.006.

33. ^ Helena Janols, Caroline Bergenfelz, Roni Allaoui, Anna-Maria Larsson, Lisa Rydén, Sven Björnsson. (2014). A high frequency of MDSCs in sepsis patients, with the granulocytic subtype dominating in gram-positive cases. Journal of Leukocyte Biology, vol. 96 (5), 685-693. doi:10.1189/jlb.5hi0214-074r.

34. ' Christopher Groth, Xiaoying Hu, Rebekka Weber, Viktor Fleming, Peter Altevogt, Jochen Utikal. (2018). Immunosuppression mediated by myeloid-derived suppressor cells (MDSCS) during tumour progression. BrJ Cancer, vol. 120 (1), 16-25. doi:10.1038/s41416-018-0333-1.

35. ^ (2020). COVID-19 More Frequent, Severe in Cancer Patients. Cancer Discov. doi:10.1158/2159-8290.cd-nb2020-032.

36. ^ Sarah LaCorte. (2020). The Impact of COVID-19 on Cancer Patients. Oncology Times, vol. 42 (8), 13. doi:10.1097/01.cot.0000661868.76700.93.

37. ^ COVID-19 Mortality Shows Strong Correlation With Active Cancer.

38. ^ Lennard Y W Lee, Jean Baptiste Cazier, T Starkey, CD Turnbull, Rachel Kerr, Gary Middleton. (2020). COVID-19 mortality in patients with cancer on chemotherapy or other anticancer treatments: a prospective cohort study. The Lancet. doi:10.1016/s0140-6736(20)31173-9.

39. ^ Peiwen Ma, Pamela L. Beatty, John McKolanis, Randal Brand, Robert E. Schoen, Olivera J. Finn. (2019). Circulating Myeloid Derived Suppressor Cells (MDSC) That Accumulate in Premalignancy Share Phenotypic and Functional Characteristics With MDSC in Cancer. Front. Immunol., vol. 10 . doi:10.3389/fimmu.2019.01401.

40. ^ Megan A. O'Connor, Jessica L. Rastad, William R. Green. (2017). The Role of MyeloidDerived Suppressor Cells in Viral Infection. Viral Immunology, vol. 30 (2), 82-97. doi:10.1089/vim.2016.0125.

41. ` Weiping Cai, Aiping Qin, Pengle Guo, Dehong Yan, Fengyu Hu, Qiong Yang. (2013). 
Clinical Significance and Functional Studies of Myeloid-Derived Suppressor Cells in Chronic Hepatitis C Patients. J Clin Immunol, vol. 33 (4), 798-808. doi:10.1007/s10875012-9861-2.

42. `Weiping Cai, Aiping Qin, Pengle Guo, Dehong Yan, Fengyu Hu, Qiong Yang. (2013). Clinical Significance and Functional Studies of Myeloid-Derived Suppressor Cells in Chronic Hepatitis C Patients. J Clin Immunol, vol. 33 (4), 798-808. doi:10.1007/s10875012-9861-2.

43. `Carmela De Santo, Mariolina Salio, S. Hajar Masri, Laurel Yong-Hwa Lee, Tao Dong, Anneliese O. Speak. (2008). Invariant NKT cells reduce the immunosuppressive activity of influenza A virus-induced myeloid-derived suppressor cells in mice and humans. J. Clin. Invest., vol. 118 (12), 4036-4048. doi:10.1172/jci36264.

44. ^ Carmela De Santo, Mariolina Salio, S. Hajar Masri, Laurel Yong-Hwa Lee, Tao Dong, Anneliese O. Speak. (2008). Invariant NKT cells reduce the immunosuppressive activity of influenza A virus-induced myeloid-derived suppressor cells in mice and humans. J. Clin. Invest., vol. 118 (12), 4036-4048. doi:10.1172/jci36264.

45. ^Aiping Qin, Weiping Cai, Ting Pan, Kang Wu, Qiong Yang, Nina Wang. (2012). Expansion of Monocytic Myeloid-Derived Suppressor Cells Dampens T Cell Function in HIV-1-Seropositive Individuals.J. Virol., vol. 87 (3), 1477-1490. doi:10.1128/jvi.0175912.

46. ^ Carmelo Carmona-Rivera, Wenpu Zhao, Srilakshmi Yalavarthi, Mariana J Kaplan. (2014). Neutrophil extracellular traps induce endothelial dysfunction in systemic lupus erythematosus through the activation of matrix metalloproteinase-2. Ann Rheum Dis, vol. 74 (7), 1417-1424. doi:10.1136/annrheumdis-2013-204837.

47. ^ Ronald H. L. Li, Fern Tablin. (2018). A Comparative Review of Neutrophil Extracellular Traps in Sepsis. Front. Vet. Sci., vol. 5. doi:10.3389/fvets.2018.00291.

48. ^ Naomi-Liza Denning, Monowar Aziz, Steven D. Gurien, Ping Wang. (2019). DAMPs and NETs in Sepsis. Front. Immunol., vol. 10 . doi:10.3389/fimmu.2019.02536.

49. ^ Marcel Levi, Jecko Thachil, Toshiaki Iba, Jerrold H Levy. (2020). Coagulation abnormalities and thrombosis in patients with COVID-19. The Lancet Haematology, vol. 7 (6), e438-e440. doi:10.1016/s2352-3026(20)30145-9.

50. ^ Kimberly Martinod, Denisa D. Wagner. (2014). Thrombosis: tangled up in NETs. doi:10.1182/blood-2013-10-463646.

51. ^ Alain Thierry, Benoit ROCH. (2020). NETs By-products and Extracellular DNA May Play a Key Role in COVID-19 Pathogenesis: Incidence on Patient Monitoring and Therapy, doi:10.20944/preprints202004.0238.v1.

52. ^ Brittany Mathias, Amber L. Delmas, Tezcan Ozrazgat-Baslanti, Erin L. Vanzant, Benjamin E. Szpila, Alicia M. Mohr. (2017). Human Myeloid-derived Suppressor Cells 
are Associated With Chronic Immune Suppression After Severe Sepsis/Septic Shock. Annals of Surgery, vol. 265 (4), 827-834. doi:10.1097/sla.0000000000001783.

53. `Wendy R. Francis, Rachel E. Ireland, Abigail M. Spear, Dominic Jenner, Sarah A. Watts, Emrys Kirkman. (2019). Flow Cytometric Analysis of Hematopoietic Populations in Rat Bone Marrow. Impact of Trauma and Hemorrhagic Shock. Cytometry, vol. 95 (11), 1167-1177. doi:10.1002/cyto.a.23903.

54. ^ Fabienne Venet, Julie Demaret, Morgane Gossez, Guillaume Monneret. (2020). Myeloid cells in sepsis-acquired immunodeficiency. Ann. N.Y. Acad. Sci.. doi:10.1111/nyas.14333.

55. ^ D. Grimaldi, F. Pène. (2019). Short-and Long-Term ICU-Acquired Immunosuppression. doi:10.1007/978-3-030-24250-3_11.

56. ^ McKenzie K. Hollen, Julie A. Stortz, Dijoia Darden, Marvin L. Dirain, Dina C. Nacionales, Russell B. Hawkins. (2019). Myeloid-derived suppressor cell function and epigenetic expression evolves over time after surgical sepsis. Crit Care, vol. 23 (1). doi:10.1186/s13054-019-2628-X.

57. ^ Naeem K Patil. (2019). Protective effect of LDK 378 during sepsis: a novel mechanism of action targeting myeloid-derived suppressor cells. Immunol Cell Biol, vol. 97 (10), 862-864. doi:10.1111/imcb.12296. 